\title{
Evolution of dispersal distance: maternal investment leads to bimodal dispersal kernels
}

\author{
Emanuel A. Fronhofer ${ }^{1,2, a}$, Hans Joachim Poethke ${ }^{1, b}$ \\ and Ulf Dieckmann ${ }^{3, c}$
}

1 Field Station Fabrikschleichach, University of Würzburg Glashüttenstrasse 5, D-96181 Rauhenebrach, Germany

2 Eawag: Swiss Federal Institute of Aquatic Science and Technology, Department of Aquatic Ecology, Überlandstrasse 133, CH-8600 Dübendorf, Switzerland

3 Evolution and Ecology Program, International Institute for Applied Systems Analysis, Schlossplatz 1, A-2361 Laxenburg, Austria
a emanuel.fronhofer@eawag.ch
b poethke@biozentrum.uni-wuerzburg.de
c dieckmann@iiasa.ac.at

Running title: Evolution of dispersal distance

Keywords: dispersal kernel, dispersal evolution, maternal investment, fat-tail, bimodality

Word count: ca. 5500 (excl. references and figure legends)

Figure count: 4 (+ supporting information S1)

Table count: 1 
2 As much dispersal research has focused on the eco-evolutionary dynamics of dispersal rates, it remains 3 unclear what shape evolutionarily stable dispersal kernels must be expected to have. Yet, detailed 4 knowledge about dispersal kernels, quantifying the statistical distribution of dispersal distances, is of ${ }_{5}$ pivotal importance for understanding biogeographic diversity, predicting species invasions, and explaining 6 range shifts. We therefore examine the evolution of dispersal kernels in an individual-based model of a 7 population of sessile organisms, such as trees or corals. Specifically, we analyze the influence of three 8 potentially important factors on the shape of dispersal kernels: distance-dependent competition, distance9 dependent dispersal costs, and maternal investment reducing an offspring's dispersal costs through a trade-off with maternal fecundity. We find that without maternal investment, competition and dispersal costs lead to unimodal kernels, with increasing dispersal costs reducing the kernel's width and tail weight. 12 Unexpectedly, maternal investment inverts this effect: kernels become bimodal at high dispersal costs. ${ }_{13}$ This increases a kernel's width and tail weight, and thus the fraction of long-distance dispersers, at the 14 expense of simultaneously increasing the fraction of non-dispersers. We finally demonstrate the qualitative 15 robustness of our results against variations in a majority of tested parameter combinations. 


\section{Introduction}

Understanding and eventually predicting the distribution of species in space and time has never been more important. Within the boundaries of local adaptation a species' range is mainly influenced by its dispersal abilities (Kokko \& López-Sepulcre, 2006). Consequently there are numerous empirical and even more theoretical studies on dispersal of plants and animals (Clobert et al., 2012), but so far, particularly theoretical studies on dispersal have mainly focused on the emigration propensity of individuals, while the dispersal process itself and the question how far to disperse has been mostly ignored or tackled with rather arbitrary assumptions like nearest neighbour (e.g. Travis et al., 1999; Gros et al., 2006) or global dispersal (e.g. Poethke \& Hovestadt, 2002). However, the growing awareness of the enormous influence of dispersal distances on colonization and range expansion particularly in plants (Nichols \& Hewitt, 1994; Nathan et al., 2002; Bohrer et al., 2005; Nathan, 2006; Alsos et al., 2007) has inspired a more thorough analysis of so-called dispersal kernels - the statistical distribution of propagules in terms of distances travelled from their origin (Cousens et al., 2008; Hovestadt et al., 2012). The specific form of such kernels defines not only the mean dispersal distance, but also the occurrence of potentially important but rare long-distance dispersal events (LDD; Kot et al., 1996; Muller-Landau et al., 2003). 'Fat-tailed' distributions, which imply a relatively large proportion of LDD, increase the velocity of species invasions (Kot et al., 1996; Caswell et al., 2003), their ability to cope with habitat fragmentation (Dewhirst \& Lutscher, 2009), and may influence biogeographic patterns of species diversity (Chave et al., 2002; Nathan, 2006).

An additional challenge arises from the term 'dispersal kernel' not always being clearly defined. A kernel may be described by two distinctly different probability-density functions (pdfs): (i) the density pdf, which describes the density of propagules to be expected at a certain distance, and (ii) the distance pdf, which describes the distribution of distances the propagules are dispersed to (see also Cousens et al., 2008; Hovestadt et al., 2012). While both definitions are correct and kernels can be expressed either way, their shapes will systematically differ. For example, if one considers a uniform distribution of propagules per area up to a certain maximal dispersal distance, the density pdf will look like a cylinder, while the distance pdf will be a linearly increasing function of distance up to the maximal dispersal distance. This is simply due to the fact that in two dimensions the area of a circle increases quadratically with its radius, so the area of a thin ring at the circle's perimeter increases linearly with its radius. Thus, if the propagule density is to be constant within each ring independent of its radius, proportionally more propagules have to be dispersed to larger distances, so as to yield the same propagule density for larger rings. Throughout this manuscript, we express dispersal kernels in terms of their distance pdf.

In spite of the immense relevance of the specific form of the dispersal kernel, it is still unclear how an optimal or evolutionarily stable kernel should look like. This question has been addressed for the first time 
by Hovestadt et al. (2001), who found that fat-tailed dispersal kernels evolve in autocorrelated landscapes (and at sufficiently fine scales, all landscapes are autocorrelated). While a certain fraction of propagules will disperse to the immediate surroundings of the parent, a significant fraction of propagules will exhibit long-distance dispersal: the latter propagules disperse more or less uniformly over the landscape, which minimizes kin competition (Hamilton \& May, 1977; Rousset \& Gandon, 2002). The shape of the dispersal kernel can thus be understood as the result of two opposing selection pressures: kin competition would be minimized by a completely uniform distribution of propagules, while distance-dependent dispersal costs (for a review, see Bonte et al., 2012) select against long-distance dispersal.

While Hovestadt et al. (2001) include dispersal costs only implicitly, via an assumption of increasingly unsuitable habitat Rousset \& Gandon (2002) explicitly analysed the effect of distance-dependent dispersal costs. They predict unimodal dispersal kernels (in terms of the distance pdf) for all scenarios with costs monotonically increasing with dispersal distance. Like most studies on dispersal evolution Rousset \& Gandon (2002) assume that dispersal is under the control of the dispersing individual. Yet, this assumption is rather unlikely to be completely true for passively dispersing propagules like seeds. Thus, Starrfelt \& Kokko (2010) have studied the evolution of dispersal distance and kernel shapes in the context of parent-offspring conflict. They could show that maternal control of dispersal generally leads to longer dispersal distances and even to fat-tailed kernels.

While all these earlier studies represent important steps towards a better understanding of the evolution of the shapes of dispersal kernels, two fundamental issues known to heavily influence dispersal evolution have not yet been taken into account: (i) effects of the overall strength, and gradual attenuation with distance, of competitive interactions have not been considered, and (ii) effects of trade-offs in parental investment into offspring dispersal have not been investigated. Firstly, as Berger et al. (2008) point out, competition is a process that fundamentally shapes the spatial patterns found in plant communities and that needs to be modeled at the individual level (see e.g. Law et al., 2003; Travis et al., 2010; North et al., 2011), and not only at the population level. A large number of models in dispersal ecology are grid-based (e.g. Murrell et al., 2002; Gros et al., 2006; Bonte et al., 2010), which implies either that competition acts at the local population level, or — if only one individual is allowed per grid cell - that the assumed competition kernel has a quadratic base, which is a somewhat artificial assumption. Secondly, if one concedes that in passive dispersers the dispersal process, more specifically the dispersal distance, is centrally influenced by the parent organism ('maternal control' as in Starrfelt \& Kokko 2010) it is also very likely that parents will invest in the dispersal abilities of their offspring (for empirical evidence see Wheelwright \& Logan, 2004). It has been shown theoretically and empirically (e.g. Roff, 1994; Fronhofer et al., 2011; Burton et al., 2010; Travis et al., 2010, 2012) that life-history trade-offs, e.g., between reproduction and dispersal ability, may deeply influence the evolution of dispersal, in a way 
that may lead, for example, to polymorphisms in which low-dispersal and high-dispersal morphs coexist. In the context of sessile organisms with passive dispersal, such trade-offs are inter-generational and are more appropriately described in terms of maternal investments that may offset an offspring's dispersal costs. Especially in plants, in which seeds are surrounded by maternal tissue and may depend on these structures for dispersal, it is sensible to include this aspect and to analyze the consequences of such maternal investment.

Therefore, we here present an individual-based model of a population of sessile organisms, such as trees or corals, and investigate the evolution of the shapes of dispersal kernels. In contrast to the great majority of existing models (e.g. Murrell et al., 2002; Gros et al., 2006; Bonte et al., 2010; North et al., 2011), we do not a priori assume any specific kernel shape. Instead, we derive evolutionarily optimal kernel shapes under the assumption that long-term evolution can find ways to realize them. We explicitly account for three different selection pressures of relevance for the evolution of the shape of dispersal kernels: distance-dependent competition (e.g., Roughgarden, 1974; Law et al., 2003; Travis et al., 2010; North et al., 2011), distance-dependent dispersal costs (for a review, see Bonte et al., 2012), and maternal investment reducing the dispersal costs experienced by dispersing offspring.

\section{The model}

In our model, each individual $(i=1, \ldots, N)$ is characterized by its location $\left(x_{i}, y_{i}\right)$ and its dispersal kernel $\left(P_{i}\right)$. Individuals are located in a two-dimensional spatially continuous and homogeneous habitat, with $0 \leq x_{i}, y_{i} \leq 100$ and periodic boundary conditions. Time is discrete and generations are overlapping.

\section{Dispersal kernels}

We define dispersal kernels as probability distributions $(P(d))$ of reaching a distance $(d)$ after a dispersal event, i.e., we use a distance pdf. Since we do not a priori restrict attention to a specific functional relationship between $P$ and $d$, the dispersal kernels in our model are implemented as function-valued traits (Dieckmann et al., 2006). As is common in studies of function-valued traits, we approximate the theoretically infinite-dimensional trait by a sufficiently large, but finite, number of values. Specifically, we use $n=21$ values to describe the probabilities of reaching a distance class $\left(d_{k}, k=1, \ldots, n\right.$, with $d_{1}$ corresponding to $d=0, d_{2}$ corresponding to $0<d \leq 1, d_{3}$ corresponding to $1<d \leq 2$, ., and with $d_{21}$ corresponding to $\left.19<d \leq 20\right)$. All values $d_{k}$ are positive and are normalized so as to sum up to 1 , $\sum_{k=1}^{n} P\left(d_{k}\right)=1$ (see also Hovestadt et al., 2001; Starrfelt \& Kokko, 2010).

To ensure that the chosen trait discretization does not unduly influence our results, we carried out additional numerical analyses with up to $n=31$ distance classes. We also analyzed the effect of increasing 
the extent of the first distance class (with $d_{1}$ corresponding, instead of to $d=0$, to $0 \leq d \leq 0.1$ or to $0 \leq d \leq 0.2)$. These robustness checks are discussed in detail below; here we only mention that our results remained essentially unchanged.

\section{Reproduction}

Once per time step, all individuals reproduce sexually. They produce a stochastic number of offspring, drawn from a Poisson distribution with mean $\lambda$. As our model is applicable, for example, to trees, we assume that individuals are simultaneously monoecious, i.e., they have male and female reproductive organs. Selfing is excluded, and for simplicity we assume that an individual mates with its nearest neighbour as in Starrfelt \& Kokko (2010).

\section{Inheritance}

As our model is phenotypic, offspring inherit for each distance class of their dispersal kernel the midparental value of their two parents, altered by a segregation kernel (Roughgarden, 1979). The latter is given by a normal distribution with the mid-parental value as mean and $\sigma_{\mathrm{s}}=0.1$ as standard deviation. This allows us to include the effects resulting from the processes of segregation and recombination during meiosis.

We additionally assume rare mutation events. The mutation rate is constant $(m=0.001)$. To optimize computing time, the root-mean-square size of mutational steps, i.e., the average amount by which a value $P_{i}\left(d_{k}\right)$ is changed by mutation decreases with time (see also Poethke et al., 2010): $\sigma_{\mathrm{m}}=e^{-5 t / t_{\max }}$, with $t$ denoting time and $t_{\max }$ the maximal time considered in a model run. To guarantee that segregation, recombination and mutations result in positive numbers for the kernel the values are log-transformed before the mid-parent values are altered by the segregation kernel and mutations. Mutations are applied after segregation, with mutational increments being drawn from a normal distribution with zero mean and standard deviation $\sigma_{\mathrm{m}}$.

\section{Dispersal}

As we investigate the evolution of the dispersal kernel of sessile organisms with passive dispersal, we assume maternal control of dispersal (see also North et al., 2011). This means that the mother's genotype defines the dispersal distance of the offspring. As Hamilton \& May (1977) note optimal dispersal distances may be different depending on whether one maximizes the inclusive fitness of the mother or of the offspring. Due to costs of dispersal applying directly to the offspring, dispersal distances under offspring control are often reduced. This has been analyzed in detail by Starrfelt \& Kokko (2010). We have run 
additional numerical analyses with offspring control and found that the results corresponded well to their results.

The dispersal distance of an offspring is determined by randomly drawing a distance class $\left(d_{k}\right)$ according to the maternal dispersal kernel $\left(P\left(d_{k}\right)\right)$. The realized dispersal distance is drawn randomly with a uniform distribution from this interval, i.e., if $d_{1}$ is drawn the dispersal distance $(d)$ is always zero, if $d_{2}$ is drawn the dispersal distance is between 0 and $1(0<d \leq 0.1)$ and so forth.

\section{Dispersal costs and maternal investment}

As we assume a constant per step mortality $\left(\mu_{d}^{0}\right)$ the probability of dying while dispersing over a given distance $(\delta)$ follows an exponential function (figure 1)

$$
\mu_{\mathrm{d}}=1-e^{-\mu_{d}^{0} \delta}
$$

Of course, the experienced dispersal costs will not depend on the net distance travelled, but on the realization of the dispersal event. Logically, equation 1 holds for a straight line walk. For any other realization the cost function will follow the general form $\mu_{d}=1-e^{-\mu_{d}^{0} \delta^{v} / c}$. If the realization is a (correlated) random walk, i.e. follows a Lévy process, we find that $v<1$ (results not shown). This does not change the shape of the dispersal cost function qualitatively: $v<1$ increases the slope of the function for small distances while it saturates later. Additional numerical analyses show that our results are not influenced qualitatively by this assumption. For a detailed analysis of the influence of different dispersal cost functions see Rousset \& Gandon (2002).

Dispersal costs may be offset — at least to some extent — by maternal investment which increases the dispersal ability of propagules. Such a trade-off implies that, although investment of resources increases one component of fitness, another component of fitness is reduced (Roff \& Fairbairn, 2007). Very often an increase in dispersal ability is correlated with a decrease in fertility (Zera \& Denno, 1997; Tanaka \& Suzuki, 1998; Roff, 2002; Roff et al., 2002).

For simplicity we will assume two extreme scenarios: (i) either the offspring carry all costs as described above (equation 1; scenario 'offspring pay') or (ii) the costs are completely covered by maternal investment (scenario 'mother pays'). To keep both scenarios comparable we determine the maternally covered, kernel-dependent, costs by summing up the distance-dependent costs over the entire kernel $\left(P\left(d_{k}\right)\right)$ for all possible distance classes $\left(d_{k}\right)$,

$$
\tau=\sum_{k=1}^{n} \mu_{\mathrm{d}}\left(d_{k}\right) P\left(d_{k}\right) .
$$

\footnotetext{
Costs trade-off with fecundity (see also Burton et al., 2010; Fronhofer et al., 2011) and the mean number
} 
of offspring is then calculated as

$$
\lambda=\lambda_{0}(1-\tau)
$$

with $\lambda_{0}=4$ as our default choice.

Note that in order to analyse the influence of relaxing this strong assumption of either 'mother pays' or 'offspring pays' we ran two classes of additional numerical analyses. Firstly, we show that if both, mother and offspring, have to pay dispersal costs our results hold up to a considerable amount of costs paid by both (supporting information S3). Secondly, we allowed the allocation of costs to be itself an evolvable trait: depending on this trait a proportion of the distance dependent dispersal costs $\left(\mu_{\mathrm{d}}^{0}\right)$ is paid by the mother according to equation 2 and the remaining costs are paid by the offspring (equation 1). For all tested parameter combinations (table 1) no intermediate cost allocation strategy evolved and the evolutionarily stable strategy was full maternal investment, i.e. 'mother pays' (see supporting information S4).

\section{Competition and mortality}

No matter whether competition is for space, light or nutrients it will always depend on inter-individual distances. In addition and in contrast to previous models that assume annual organisms with nonoverlapping generations (e.g. Travis et al., 2010; North et al., 2011) we include age-dependence since competition will be asymmetric between established trees and seedlings, for example. In our model, competition acts by increasing mortality, and not by decreasing fertility (figure 1). This allows us to derive a density-dependent individual mortality term, i.e. the probability of dying in the present time step $\left(\mu_{i}\right)$. For the form of this competition kernel - often termed zone or sphere of influence (for a review of modeling approaches, see Berger et al., 2008) — we assume a general and very flexible functional relationship (analyzed in detail by Roughgarden, 1974). This approach is similar to the sphere of influence model presented by Schiffers et al. (2011). The effect of an individual $j$ on the focal individual $i$ is calculated as

$$
\mu_{i j}=e^{-\left(\Delta_{i j} / f\right)^{\gamma}} \frac{a_{j}}{a_{j}+H_{a}}
$$

with $f=\sigma \sqrt{\Gamma(1 / \gamma)} / \sqrt{\Gamma(3 / \gamma)}$, where $\Gamma$ is the gamma function. The first term of the function reflects distance- and the second term age-dependence. $\Delta_{i j}$ is the Euclidean distance between individuals $i$ and j. $\sigma$ is the standard deviation of the competition kernel and $\gamma$ determines its kurtosis: $\gamma=2$ yields a normal (mesokurtic) distribution, $\gamma<2$ a leptokurtic distribution (narrow peak and fat tails), and $\gamma>2$ a platykurtic distribution (wide peak and thin tails). The kurtosis parameter (with $\gamma=2$ as our default choice) thus determines the balance between localized competition and long-range competition, while the standard deviation (with $\sigma=1$ as our default choice) scales the width of the competition kernel. 
Age-dependence is a simple asymptotic function with $a$ as the age of tree $j$ and $H_{\mathrm{a}}$ as the halfsaturation constant, i.e. the age at which a tree reaches half of its maximal competitive ability (with $H_{\mathrm{a}}=3$ as our default choice). Note that the age-dependent term is important since without it competition between adults and seedlings is symmetric.

The total competition related mortality $\left(\mu_{i}\right)$ of individual $i$ may additionally include a baseline mortality $\left(\mu_{0}=0.1\right.$ as a standard value) which is density independent:

$$
\mu_{i}=1-\left(1-\mu_{0}\right) \prod_{i \neq j}\left(1-\mu_{i j}\right)
$$

Please see figure 1 for a summary of all mortality components. This figure includes the effects of all individuals in a landscape as in equation 5 and additionally illustrates the dominant effect of the focal individual.

\section{Numerical analyses}

All analyses were run in a world of 100 x 100 distance units with periodic boundary conditions. Depending on parameter combinations populations sizes varied roughly between 400 and over 7000 individuals. Computing time was set to $t_{\max }=10,000$ time steps, a time span that allowed all model runs to reach equilibrium. The results shown below are means over 25 replicates. Please see table 1 for a summary of relevant parameters and tested values.

\section{Results}

\section{Evolution of dispersal kernels}

In all scenarios without maternal investment, i.e. when the offspring pay distance-dependent dispersal costs according to equation 1, we find that the evolutionarily stable dispersal kernel is unimodal (figure $2 \mathrm{~A}$ ). This results from the interaction between the competition kernel, competition with related dispersers and dispersal costs (see figure 1). Increasing dispersal costs lead to narrower, more peaked and less fat-tailed kernels (figure $2 \mathrm{~B}$ ). This is due to an increase in the slope of the dispersal cost function (figure 1).

\section{Maternal investment leads to bimodal kernels}

In general, maternal investment increases the occurrence of LDD, i.e. the weight of the kernel's tail (figure 2). Interestingly, maternal investment inverts the effect of dispersal costs on long-distance dispersal: 
here increasing dispersal costs lead to an increase in tail weight (figure 2 D). In addition, the evolutionarily stable dispersal kernel for high dispersal costs is bimodal (figure 2 D) with an important proportion of propagules remaining very close to the maternal individual and a mass of offspring showing LDD. The inset in figure $2 \mathrm{D}$ shows the same numerical analysis for an increased resolution of the dispersal kernel (distance class extent of 0.5 instead of the default of 1 ). Note that the bimodality we describe here occurs at the individual level and is not the result of a mixed strategy with coexisting short- and long-distance dispersers (supporting information $\mathrm{S} 4$ ).

\section{Dispersal costs}

A more detailed analysis of the influence of dispersal costs shows that, as one would assume, the mean dispersal distance decreases with costs in the 'offspring pay' scenario (figure 3 A). Yet, with maternal investment ('mother pays') the relationship becomes u-shaped, i.e. higher dispersal costs favour higher mean dispersal distances (figure $3 \mathrm{~A}$ ). Clearly, this is due to the asymmetry and tail weight of the kernels (figure 2) since the median dispersal distance decreases monotonically with dispersal costs (figure 3 B). The median reaches a steady value which is defined by the width of the competition kernel (see also figure 4). The scenario assumed, i.e. distance costs paid by the offspring ('offspring pay') vs. kernel costs paid by the mother ('mother pays'), does not influence the median dispersal distance.

As mentioned above, increasing dispersal costs lead to narrower kernels if the offspring pay distance dependent dispersal costs (figure $3 \mathrm{C}$ ). However, in the case of maternal investment this tendency is reversed for sufficiently high dispersal costs (figure $3 \mathrm{C}$; here $\mu_{\mathrm{d}}^{0}>0.2$ ) which is due to the above described bimodality (figure $2 \mathrm{D})$.

A similar pattern can be observed for tail weight (figure $3 \mathrm{D}$ ). In the 'offspring pay' scenario increasing dispersal costs reduce tail weight, here measured as the 95 th percentile of the kernel. Maternal investment inverts this pattern: as soon as the kernel becomes bimodal its tail weight increases with dispersal costs (figure $3 \mathrm{D})$.

\section{Shape of the competition kernel}

The qualitative results presented above, i.e. the emergence of fat-tailed and bimodal kernels in scenarios with maternal investment, are robust against variation in all model parameters (figure 4 and supporting information S1). Not surprisingly, the width of the competition kernel influences the mean and the median of the dispersal kernel, i.e. the location of the peak (figure $4 \mathrm{~A}, \mathrm{~B}$ ). Wider competition kernels, i.e., higher values of the standard deviation $(\sigma)$, lead to bimodal dispersal kernels at lower dispersal costs in the 'mother pays' scenario (figure $4 \mathrm{C}, \mathrm{D}$ ). The pattern is not lost for narrower competition kernels, 
yet requires higher dispersal costs to emerge ( $\mu_{\mathrm{d}}^{0}>0.5$; not shown).

The kurtosis of the competition kernel $(\gamma)$ has only very slight effects. More leptokurtic shapes lead to slightly smaller dispersal distances (figure $4 \mathrm{E}, \mathrm{F}$ ) because the costs inflicted by competition decrease at a faster rate at small distances. As a consequence more platykurtic competition kernels lead to bimodality in 'mother pays' scenarios at lower dispersal costs (figure $4 \mathrm{G}$ ) and to more pronounced fat tails (figure $4 \mathrm{H}$ ).

The shape, especially the height of the competition kernel, also depends on the focal individual's age (equation 4). We find that the slower an individual's competitive ability increases ('slow growth' in figure 4; larger values of the half-saturation constant $H_{\mathrm{a}}$ ) the smaller dispersal distances become (figure $4 \mathrm{I}$, J). Under such conditions the usual pattern of higher dispersal distances in scenarios with maternal investment is inverted (figure $4 \mathrm{~J}$ ) which is due to a more pronounced bimodality (figure $4 \mathrm{~K}$ ) with a higher peak at distance zero. This is primarily due to the lower competitive ability of a focal maternal individual. In turn, this pattern interacts with increasing dispersal costs and allows the emergence of a heavier tail (figure $4 \mathrm{~L}$; black and grey solid lines intersect). Maternal investment allows to increase the amount of LDD, i.e. tail weight, by increasing the height of the peak at distance zero, i.e. the number of non-dispersers (see also equation 2).

\section{Discussion}

It has been recognized that in sessile organisms dispersal distance is mainly controlled by the maternal individual (see Starrfelt \& Kokko, 2010, for an analysis of parent-offspring conflict). It is less well appreciated that the mother must also invest in the dispersal ability of its offspring (e.g. Wheelwright \& Logan, 2004). Such an investment will necessarily come at a cost, i.e it will trade off with fertility or survival. It has been shown in other contexts that trade-offs may shape the evolution of dispersal considerably (e.g. Roff, 1994; Burton et al., 2010; Travis et al., 2010; Fronhofer et al., 2011; Travis et al., 2012). We here demonstrate for sessile organisms that trading fecundity for an increased survival of dispersing offspring, i.e. maternal investment, characteristically influences the form of the dispersal kernel. Particularly for high dispersal costs bimodal kernels emerge and, at the same time, the kernels become heavily fat-tailed. In contrast to previous studies that already demonstrate the evolution of bimodal distance pdfs (Starrfelt \& Kokko, 2010) our model demonstrates the evolution of bimodality in both density and distance pdfs. These results prove to be robust against variation in all tested model parameters (figures 4 and supporting information). They even hold if the offspring were to pay a part of dispersal costs themselves (supporting information S3).

By relaxing the somewhat arbitrary assumption of a world with discrete habitat patches and modelling 
inter-individual competition explicitly at the individual level in continuous space through a competition kernel, our results represent an important step towards a better understanding of dispersal in sessile organisms such as plants. Our results from scenarios in which offspring pay distance-dependent dispersal costs (figure $2 \mathrm{~A}, \mathrm{~B}$ ) are in good accordance with findings from Rousset \& Gandon (2002). Yet, they show some distinct differences as our model assumes distance-dependent competition (competition kernels), maternal control of dispersal and overlapping generations. Due to decreasing kin competition and increasing (saturating) dispersal costs Rousset \& Gandon (2002) also predict unimodal dispersal kernels in two-dimensional landscapes. By explicitly introducing competition for space we can show that the width of the competition kernel $(\sigma)$ will determine the location of the kernel's peak. Depending on their specific shape competition kernels may even increase tail weight since competitive interactions are not limited to the size of an arbitrarily defined grid-cell. Generally, our kernels are slightly more fat-tailed as we assume maternal control of dispersal (Starrfelt \& Kokko, 2010) in comparison to Rousset \& Gandon (2002). Finally, when offspring pay dispersal costs our model predicts that all offspring will disperse (figure $2 \mathrm{~A}$, B; the first distance class has a zero value) because the competition kernel drives all seeds away from the maternal individual. This will not necessarily hold for grid based models, especially if one grid cell may contain more than one individual. Rousset \& Gandon (2002) for example, do predict a certain amount of non-dispersers. This effect is especially strong, as the modelled organisms are assumed to be annual in contrast to our model. For the same reasons Starrfelt \& Kokko (2010) do not find unimodal kernels.

In scenarios with maternal investment bimodality of the dispersal kernel emerges mainly because of two mechanisms: (i) as the mother pays dispersal costs defined by the dispersal kernel (equation 2) increasing the variance of the kernel through a bimodal distribution with a peak at zero (or at very small distances) allows the mother to decrease the costs while keeping the mean dispersal distance constant or even increasing it through a heavily fat tail. Thus, by reducing the dispersal distances of some offspring the mother may achieve LDD for other propagules. Higher dispersal distances are evolutionarily advantageous because they minimize kin competition (Hamilton \& May, 1977; Rousset \& Gandon, 2002) an effect known to be especially pronounced for maternal control of dispersal (Hamilton \& May, 1977; Starrfelt \& Kokko, 2010) . (ii) Furthermore, the maternal location has an interesting attribute that makes it attractive for seed deposition. In case of the mother's death it characteristically implies a minimal influence of the nearest neighbours' competition kernels (see figure 1, thin black line). Locally it maximizes an individual's survival and non-dispersers will be able to inherit this locally optimal location ('territorial inheritance') after the mother's death.

It is immediately clear that the latter mechanism will be critically dependent on the assumed width of the first distance class. We therefore tested the impact of increasing the extent of this first class. Our 
tests show that the bimodality reported here does indeed vanish for coarse grids but is stable for a width of the first distance class $\left(d_{1}\right)$ of up to $0 \leq d \leq 0.2$ (see also figure 1 ). This results underlines the possible artifacts resulting from grid-based models in general.

The two mechanisms discussed above also explain the influence of the age-specific component of the competition kernel (figure 4) and of fecundity (see supporting information S1). Increased local competition through fast growth leads to a reduction of kernel bimodality in scenarios with maternal investment. Although there is an advantage for mothers keeping a fraction of offspring close by for territorial inheritance fast growth leads to an important increase in local competition between the mother and her non-dispersing offspring and between these. This leads to a reduction to the peak at distance zero, i.e. the number of non-dispersers. The same effect will emerge from increased numbers of offspring. As the kernel costs resulting from the formation of a fat-tail are offset by the production of 'cheaper' non-dispersers, reducing the latter will lead to a reduction of tail weight.

Due to asymmetric competition between the mother and the offspring a majority of non-dispersers may actually die. This of course begs the question why mothers produce these offspring in the first place. If this was just a strategy to spare resources for the production of more expensive long-distance dispersers and these 'cheap' propagules were thus only produced to reduce the costs of offspring production, mothers could as well simply reduce the number of offspring produced and put all resources into long-distance dispersers. In order to explore this question we ran additional numerical analyses including evolvable fecundities and a trade-off between fecundity and baseline mortality (supporting information S5 and S6). Individuals reducing their fecundity could thus increase their survival. In all these model runs, the bimodality was evolutionarily stable (supporting information S5). Regardless of the form of the assumed trade-off function (concave, linear, convex) we observed evolution towards short lived, sometimes annual, but very fecund individuals showing an increasingly bimodal kernel (supporting information S6). Dytham \& Travis (2006) analyse the concurrent evolution of longevity and dispersal distance. Their results show that shorter life spans lead to shorter dispersal distances. Although our model is significantly different, the increasing bimodality, i.e. the higher frequency of non-dispersers we find is a similar phenomenon. It results from reduced kin competition and the possibility of territorial inheritance. Yet, as the form of our kernel may evolve freely, a reduced life span does not lead to smaller dispersal distances in general. On the contrary, as noted above, in scenarios with maternal investment the kernels are bimodal and heavily fat-tailed with an important fraction of long-distance dispersers.

Interestingly the occurrence of a bimodal kernel that generates non-dispersers and long-distance dispersers parallels the co-occurrence of philopatrics and dispersers found in models of actively moving organisms that include a trade-off between fertility and dispersal ability (e.g. Roff, 1994; Fronhofer et al., 2011). Yet, as these models assume offspring control of dispersal and consider emigration rates only, the 
underlying mechanisms are different (see also below). In recent work that incorporates such a trade-off into an explicit movement model Travis et al. (2012) show that emigration rates increase for high levels of investment. Although we also find more LDD in our model emigration rates actually decrease which is due to the kernel's bimodality.

In general, maternal investment, i.e. trading fecundity for an increased survival of dispersing offspring, is highly advantageous. It allows the persistence of stable populations despite low fecundities, high mortalities and high competition, conditions that otherwise lead to global extinctions. This can be seen, e.g., in the supporting information figure S1 A-D: for low fecundities and if the offspring pay distance dependent dispersal costs populations are not viable for dispersal costs $\mu_{\mathrm{d}}^{0}>0.3$. This is not the case in scenarios with maternal investment. Additional numerical analyses show that maternal investment allows populations to survive dispersal costs over 0.8 (not shown). Simulation experiments also show that the 'mother pays' strategy is evolutionarily stable (supporting information S4).

To summarize so far and put our results in a broader context, if the offspring control dispersal the evolutionarily stable kernel must guarantee equal fitness expectations for all offspring (see Rousset \& Gandon, 2002). This logic does not apply if dispersal is controlled maternally as we assume in this study: the parent-offspring conflict (discussed in the context of dispersal distances in Starrfelt \& Kokko, 2010) leads to larger dispersal distances, as the maternal individual maximizes its own inclusive fitness expectations by reducing kin competition. If the offspring pay dispersal costs, these costs are the only mechanism that restrain dispersal distance and counteract the effect of (kin) competition which drives seeds away from the maternal individual. This cost-benefit calculation is fundamentally changed if the mother also pays dispersal costs. Increasing dispersal costs lead to selection for non-dispersers in spite of strong competition with the maternal individual, as this allows the mother to reduce its investment in propagule dispersal ability, which in turn allows for more LDD. Simultaneously, selection seems to favour a reduction in longevity for an increase in fecundity. Ultimately, this may lead to highly fertile annual organisms with strongly bimodal dispersal kernels (see supporting information S5 and S6).

\section{Examples}

Our model is applicable to sessile organisms such as plants. Most plants will show maternal investment, at least to some degree, since fruit and seed production is obviously maternally regulated. The bimodal dispersal kernel which we predict can be realized for example by seed polymorphisms, a phenomenon that has been frequently observed (for a review, see Imbert, 2002). The Asteraceae Heterotheca latifolia for example shows a dimorphism in achene structure: while disc achenes, which have a pappus, are wind dispersed and responsible for LDD ray achenes are not (Venable \& Levin, 1985). This polymorphic seed structure will lead to bimodality in the dispersal kernel and increased tail weight (see also van Mölken 
et al., 2005; Brändel, 2007). The same effect can be achieved by polychory, i.e. the use of more than one seed dispersal agent (e.g., Berg, 1966; Jordano et al., 2007; Russo et al., 2006). For example in Prunus mahaleb small birds are responsible for short distance dispersal while fruits eaten by mammals and larger birds are dispersed over long distances (Jordano et al., 2007). In addition our results are in good accordance with the increasing evidence that multiple seed size strategies, directly leading to different dispersal distances, may generally be due to life-history trade-offs (competition-colonization or stress tolerance-fecundity trade-offs, see e.g. Jakobsson \& Eriksson, 2003; Lönnberg \& Eriksson, 2013). Of course, in purely wind-dispersed plants with monomorphic seeds such as e.g. modelled by Travis et al. (2010) trade-offs may also occur e.g. between plant height which influences dispersal distance and fertility. Such trade-offs may then lead to the evolution of different plant heights depending e.g. on habitat availability or local extinctions.

In a very broad sense our results may be of significance for territorial animals, although the model has not been designed for actively moving animals. Of course, in this case competition will be more prone to act on fertility than on mortality, but the resulting patterns may be similar. A dimorphism can often be observed between non-dispersers that wait, eventually help their parents, and bet on territorial inheritance and dispersers that try to colonize new, eventually empty territories (e.g. Kokko \& Ekman, $2002)$.

\section{Simplifications}

As in any tractable model we include some simplifying assumptions, a number of which we have already dealt with throughout this paper. A central simplification we have made is that dispersal strategies, i.e., the dispersal kernel, are not age-dependent, although we assume overlapping generations. Maternal age-dependent dispersal has been analyzed by Ronce et al. (1998) who provide theoretical and empirical evidence that such a strategy is evolutionarily advantageous, for both maternal and offspring control of dispersal. As we have discussed above the emerging bimodal dispersal kernel is a result of cost optimization in order to allow LDD and at the same time insures territorial inheritance. Yet, these two aspects are advantageous respectively early and late in the life of an individual. We hypothesize that age-dependent kernels would be fat-tailed in early life stages and more biased towards short-distance dispersal and the production of non-dispersing offspring later on in order to reduce (kin) competition but still allow territorial inheritance.

Evidently, dispersal could also occur through time (dormancy). Trade-offs and correlations between dormancy, longevity and spatial dispersal have been analysed theoretically and empirically (e.g. Rees, 1993). These additional complexities are far beyond the scope of this article and will not be discussed in detail here. Note that dormancy as a bet-hedging strategy is especially relevant in variable environments 
(for a review see e.g. Childs et al., 2010) that are not in the focus of this study.

Obviously, the uniformity and stability of the landscape we assume here is a further simplification. Although space is continuous in our model, it is homogeneous and shows no habitat structure or turnover. As Hovestadt et al. (2001) predict fat-tailed dispersal kernels to emerge in autocorrelated landscapes, we are confident that the introduction of spatial structure would not alter our results fundamentally (for a detailed treatment of the influence of habitat structure on dispersal distance, see North et al., 2011). Of course, patch size would interact with the competition kernel and influence the evolving mean dispersal distance and the location of the dispersal kernel's maximum or second peak for bimodal kernels. Yet, as the introduction of suitable and non-suitable habitat basically leads to an increase in dispersal costs we hypothesize that spatial structure would only underline our results for both scenarios. Especially in scenarios with maternal investment the bimodality of the dispersal kernel should be more pronounced, provided that patches are large enough to support more than one individual. A bimodal kernel is highly advantageous in fragmented landscapes with patch turnover as well, since the fat tail and resulting LDD allows individuals to colonize distant and empty patches. At the same time the non- and short distancedispersers emerging from the same kernel in the next generation guarantee a successful and sustained establishment (see also North et al., 2011).

\section{Conclusions}

Although the concept of a dispersal kernel is not new only little work has been done on the evolution of the shape of dispersal kernels. In contrast to previous work (Hovestadt et al., 2001; Rousset \& Gandon, 2002; Starrfelt \& Kokko, 2010) we have concentrated on two important aspects that have received little attention in this context up to now: (i) the effects of individual competition kernels in continuous space and (ii) maternal investment. We predict the emergence of heavily fat-tailed and bimodal dispersal kernels for sessile organism with overlapping generations.

\section{Acknowledgements}

The authors thank Thomas Hovestadt and Alex Kubisch for inspiring discussions and Andreas Gros for exploratory numerical work. E.A.F. was supported by a grant of the German Excellence Initiative of the Graduate School of Life Sciences, University of Würzburg. U.D. gratefully acknowledges financial support by the European Commission, the European Science Foundation, the Austrian Science Fund, the Austrian Ministry for Science and Research, and the Vienna Science and Technology Fund. 


\section{Literature cited}

Alsos, I.G., Eidesen, P.B., Ehrich, D., Skrede, I., Westergaard, K., Jacobsen, G.H., Landvik, J.Y., Taberlet, P. \& Brochmann, C. 2007. Frequent long-distance plant colonization in the changing arctic. Science 316: 1606-1609.

Berg, R.Y. 1966. Seed dispersal of Dendromecon — its ecologic evolutionary and taxonomic significance. Am. J. Bot. 53: 61-73.

Berger, U., Piou, C., Schiffers, K. \& Grimm, V. 2008. Competition among plants: concepts, individualbased modelling approaches, and a proposal for a future research strategy. Perspect. Plant Ecol. Evol. Syst. 9: 121-135.

Bohrer, G., Nathan, R. \& Volis, S. 2005. Effects of long-distance dispersal for metapopulation survival and genetic structure at ecological time and spatial scales. J. Ecol. 93: 1029-1040.

Bonte, D., Hovestadt, T. \& Poethke, H.J. 2010. Evolution of dispersal polymorphism and local adaptation of dispersal distance in spatially structured landscapes. Oikos 119: 560-566.

Bonte, D., Van Dyck, H., Bullock, J.M., Coulon, A., Delgado, M., Gibbs, M., Lehouck, V., Matthysen, E., Mustin, K., Saastamoinen, M., Schtickzelle, N., Stevens, V.M., Vandewoestijne, S., Baguette, M., Bartoń, K., Benton, T.G., Chaput-Bardy, A., Clobert, J., Dytham, C., Hovestadt, T., Meier, C.M., Palmer, S.C.F., Turlure, C. \& Travis, J.M.J. 2012. Costs of dispersal. Biol. Rev. 87: 290-312, URL http://dx.doi.org/10.1111/j.1469-185X.2011.00201.x.

Brändel, M. 2007. Ecology of achene dimorphism in Leontodon saxatilis. Ann. Bot. 100: 1189-1197.

Burton, O.J., Pillips, B.L. \& Travis, J.M.J. 2010. Trade-offs and the evolution of life-histories during range expansion. Ecol. Lett. 13: 1210-1220.

Caswell, H., Lensink, R. \& Neubert, M.G. 2003. Demography and dispersal: Life table response experiments for invasion speed. Ecology 84: 1968-1978.

Chave, J., Muller-Landau, H.C. \& Levin, S.A. 2002. Comparing classical community models: Theoretical consequences for patterns of diversity. Am. Nat. 159: 1-23.

Childs, D.Z., Metcalf, C.J.E. \& Rees, M. 2010. Evolutionary bet-hedging in the real world: empirical evidence and challenges revealed by plants. Proc. R. Soc. B-Biol. Sci. 277: 3055-3064, URL http://rspb.royalsocietypublishing.org/content/277/1697/3055. abstract.

Clobert, J., Baguette, M., Benton, T.G. \& Bullock, J.M. 2012. Dispersal Ecology and Evolution. Oxford University Press, Oxford, UK. 
Cousens, R., Dytham, C. \& Law, R. 2008. Dispersal in Plants - A Population Perspective. Oxford University Press, Oxford, UK.

Dewhirst, S. \& Lutscher, F. 2009. Dispersal in heterogeneous habitats: thresholds, spatial scales, and approximate rates of spread. Ecology 90: 1338-1345.

Dieckmann, U., Heino, M. \& Parvinen, K. 2006. The adaptive dynamics of function-valued traits. J. Theor. Biol. 241: 370-389.

Dytham, C. \& Travis, J.M.J. 2006. Evolving dispersal and age at death. Oikos 113: 530-538.

Fronhofer, E.A., Kubisch, A., Hovestadt, T. \& Poethke, H.J. 2011. Assortative mating counteracts the evolution of dispersal polymorphisms. Evolution 65: 2461-2469.

Gros, A., Poethke, H.J. \& Hovestadt, T. 2006. Evolution of local adaptations in dispersal strategies. Oikos 114: 544-552.

Hamilton, W.D. \& May, R.M. 1977. Dispersal in stable habitats. Nature 269: 578-581.

Hovestadt, T., Bonte, D., Dytham, C. \& Poethke, H.J. 2012. Evolution and emergence of dispersal kernels - a brief theoretical evaluation. In: Dispersal Ecology and Evolution (J. Clobert, M. Baguette \& T.G. Benton, eds), pp. 211-221. Oxford University Press.

Hovestadt, T., Messner, S. \& Poethke, H.J. 2001. Evolution of reduced dispersal mortality and 'fat-tailed' dispersal kernels in autocorrelated landscapes. Proc. R. Soc. B-Biol. Sci. 268: 385-391.

Imbert, E. 2002. Ecological consequences and ontogeny of seed heteromorphism. Perspect. Plant Ecol. Evol. Syst. 5: 13-36.

Jakobsson, A. \& Eriksson, O. 2003. Trade-offs between dispersal and competitive ability: a comparative study of wind-dispersed asteraceae forbs. Evol. Ecol. 17: 233-246.

Jordano, P., Garcia, C., Godoy, J.A. \& Garcia-Castano, J.L. 2007. Differential contribution of frugivores to complex seed dispersal patterns. Proc. Natl. Acad. Sci. U. S. A. 104: 3278-3282.

Kokko, H. \& Ekman, J. 2002. Delayed dispersal as a route to breeding: Territorial inheritance, safe havens, and ecological constraints. Am. Nat. 160: 468-484.

Kokko, H. \& López-Sepulcre, A. 2006. From individual dispersal to species ranges: Perspectives for a changing world. Science 313: 789-791.

Kot, M., Lewis, M.A. \& van den Driessche, P. 1996. Dispersal data and the spread of invading organisms. Ecology 77: 2027-2042. 
Law, R., Murrell, D.J. \& Dieckmann, U. 2003. Population growth in space and time: Spatial logistic equations. Ecology 84: 252-262.

Lönnberg, K. \& Eriksson, O. 2013. Rules of the seed size game: contests between large-seeded and small-seeded species. Oikos 122: 1080-1084, URL http://dx.doi.org/10.1111/j.1600-0706.2012.00249.x.

Muller-Landau, H.C., Levin, S.A. \& Keymer, J.E. 2003. Theoretical perspectives on evolution of longdistance dispersal and the example of specialized pests. Ecology 84: 1957-1967.

Murrell, D.J., Travis, J.M.J. \& Dytham, C. 2002. The evolution of dispersal distance in spatiallystructured populations. Oikos 97: 229-236.

Nathan, R. 2006. Long-distance dispersal of plants. Science 313: 786-788.

Nathan, R., Katul, G.G., Horn, H.S., Thomas, S.M., Oren, R., Avissar, R., Pacala, S.W. \& Levin, S.A. 2002. Mechanisms of long-distance dispersal of seeds by wind. Nature 418: 409-413.

Nichols, R.A. \& Hewitt, G.M. 1994. The genetic consequences of long-distance dispersal during colonization. Heredity 72: 312-317.

North, A., Cornell, S. \& Ovaskainen, O. 2011. Evolutionary responses of dispersal distance to landscape structure and habitat loss. Evolution 65: 1739-1751.

Poethke, H.J. \& Hovestadt, T. 2002. Evolution of density- and patch-size-dependent dispersal rates. Proc. R. Soc. B-Biol. Sci. 269: 637-645.

Poethke, H.J., Weisser, W.W. \& Hovestadt, T. 2010. Predator-induced dispersal and the evolution of conditional dispersal in correlated environments. Am. Nat. 175: 577-586.

Rees, M. 1993. Trade-offs among dispersal strategies in british plants. Nature 366: 150-152, URL http://dx.doi.org/10.1038/366150a0.

Roff, D.A. 1994. Habitat persistence and the evolution of wing dimorphism in insects. Am. Nat. 144: 772-798.

Roff, D.A. 2002. Life History Evolution. Sinauer Associates, Sunderland, MA.

Roff, D.A. \& Fairbairn, D.J. 2007. The evolution of trade-offs: Where are we? J. Evol. Biol. 20: 433-447.

Roff, D.A., Mostowy, S. \& Fairbairn, D.J. 2002. The evolution of trade-offs: Testing predictions on response to selection and environmental variation. Evolution 56: 84-95. 
Ronce, O., Clobert, J. \& Massot, M. 1998. Natal dispersal and senescence. Proc. Natl. Acad. Sci. U. S. A. 95: 600-605.

Roughgarden, J. 1974. Species packing and competition function with illustrations from coral-reef fish. Theor. Popul. Biol. 5: 163-186.

Roughgarden, J. 1979. Theory of Population Genetics and Evolutionary Ecology: An Introduction. Macmillan Publishing Co., Inc.

Rousset, F. \& Gandon, S. 2002. Evolution of the distribution of dispersal distance under distancedependent cost of dispersal. J. Evol. Biol. 15: 515-523.

Russo, S.E., Portnoy, S. \& Augspurger, C.K. 2006. Incorporating animal behavior into seed dispersal models: Implications for seed shadows. Ecology 87: 3160-3174.

Schiffers, K., Tielbörger, K., Tietjen, B. \& Jeltsch, F. 2011. Root plasticity buffers competition among plants: theory meets experimental data. Ecology 92: 610-620.

Starrfelt, J. \& Kokko, H. 2010. Parent-offspring conflict and the evolution of dispersal distance. Am. Nat. 175: $38-49$.

Tanaka, S. \& Suzuki, Y. 1998. Physiological trade-offs between reproduction, flight capability and longevity in a wing-dimorphic cricket, Modicogryllus confirmatus. J. Insect Physiol. 44: 121-129.

Travis, J.M.J., Murrell, D.J. \& Dytham, C. 1999. The evolution of density-dependent dispersal. Proc. R. Soc. B-Biol. Sci. 266: 1837-1842.

Travis, J.M.J., Mustin, K., Bartoń, K.A., Benton, T.G., Clobert, J., Delgado, M.M., Dytham, C., Hovestadt, T., Palmer, S.C.F., Van Dyck, H. \& Bonte, D. 2012. Modelling dispersal: an eco-evolutionary framework incorporating emigration, movement, settlement behaviour and the multiple costs involved. Methods in Ecology and Evolution 3: 628-641, URL http://dx.doi.org/10.1111/j.2041-210X.2012.00193.x.

Travis, J.M.J., Smith, H.S. \& Ranwala, S.M.W. 2010. Towards a mechanistic understanding of dispersal evolution in plants: conservation implications. Divers. Distrib. 16: 690-702.

van Mölken, T., Jorritsma-Wienk, L.D., Van Hoek, P.H.W. \& De Kroon, H. 2005. Only seed size matters for germination in different populations of the dimorphic Tragopogon pratensis subsp. pratensis (Asteraceae). Am. J. Bot. 92: 432-437.

Venable, D.L. \& Levin, D.A. 1985. Ecology of achene dimorphism in Heterotheca latifolia. 1. Achene structure, germination and dispersal. J. Ecol. 73: 133-145. 
Wheelwright, N.T. \& Logan, B.A. 2004. Previous-year reproduction reduces photosynthetic capacity and slows lifetime growth in females of a neotropical tree. Proc. Natl. Acad. Sci. U. S. A. 101: 8051-8055.

Zera, A.J. \& Denno, R.F. 1997. Physiology and ecology of dispersal polymorphism in insects. Annu. Rev. Entomol. 42: 207-230.

\section{Table and figure captions}

\section{Table 1:}

Important model parameters, their meaning and tested values.

\section{Figure 1:}

The components of mortality. Dispersal costs $\left(\mu_{d}\right.$, dashed line) increase asymptotically with distance (equation 1, function shown for 'offspring pay' scenario). In addition we assume a distance- and densityindependent, constant baseline mortality $\left(\mu_{0}\right.$, dotted line). The shape of the competition kernel, is very flexible and can vary from leptokurtic to platykurtic $(\gamma$, see equation 4 and text for details, thick black line). Competition with other individuals than the focal individual (e.g. a mother tree) shows a minimum at the location of the focal individual (thin black line; data taken from from numerical analyses; smooth spline regression over the mean of 100 focal individuals of age $a=3$ for each of 20 replicate simulation runs; smoothing parameter: $\lambda=0.3$ ). The resulting total mortality a dispersing propagule experiences is depicted in grey. Note that mortality is a probability and the components therefore cannot be summed up directly. Parameters: $\gamma=2, \sigma=1, a=3, H_{\mathrm{a}}=3, \mu_{\mathrm{d}}^{0}=0.1, \lambda_{0}=4$ (for the numerical analyses).

\section{Figure 2:}

Maternal investment and the evolution of dispersal kernels. All four panels show evolutionarily stable dispersal kernels (distance pdfs). The upper row (A, B) depicts the influence of dispersal costs without maternal investment, i.e. when offspring pay distance-dependent dispersal costs according to equation 1. The competition kernel (equation 4) leads to unimodal and fat-tailed distributions (A). Increasing dispersal costs lead to narrower and more peaked kernels (B). The lower row (C, D) shows kernels for scenarios with maternal investment, i.e. the mother pays kernel-dependent dispersal costs (equation 2) and reduces her fecundity in order to maximize offspring survival during dispersal (equation 3). Maternal investment leads to heavily fat-tailed kernels and to bimodality at high dispersal costs (D). The inset in panel D shows the same numerical analysis for an increased resolution of the dispersal kernel (distance class extent of 0.5 instead of the default of 1$)$. Parameters: $\lambda_{0}=4, \mu_{0}=0.1, \gamma=2, \sigma=1, H_{\mathrm{a}}=3$, and $\mu_{\mathrm{d}}^{0}=0.1$ 
(left panels; A, C) or $\mu_{\mathrm{d}}^{0}=0.4$ (right panels; B, D).

\section{Figure 3:}

Maternal investment and dispersal costs. The graphs represent a systematic analysis of the influence of dispersal costs $\left(\mu_{d}^{0}\right)$ on mean $(\mathrm{A})$, median $(\mathrm{B})$, interquartile range $(\mathrm{C})$ and the position of the 95 th percentile (D) of the evolutionarily stable dispersal kernels. Here and in the following figures the solid line represents results for scenarios with maternal investment ('mother pays') and the dashed line without ('offspring pay'). For 'mother pays' scenarios the mean dispersal distance shows a u-shaped relation with increasing dispersal costs while the median does not $(\mathrm{A}, \mathrm{B})$. The interquartile range $(\mathrm{C})$ captures the emerging bimodality in 'mother pays' scenarios. The 95th percentile is a good indicator for fat tails (D). Parameter values: $\lambda_{0}=4, \mu_{0}=0.1, \gamma=2, \sigma=1, H_{a}=3$. The grey crosses represent data points and the lines are smooth spline regressions (smoothing parameter: $\lambda=0.3$ ).

\section{Figure 4:}

Influence of the shape of the competition kernel. The competition kernel (figure 1 and equation 4) is determined by its width (standard deviation $\sigma$; panels $\mathrm{A}-\mathrm{D})$, by its kurtosis ( $\gamma$, panels $\mathrm{E}-\mathrm{H}$ ) and by its height, which is a function of the focal individual's age (half-saturation constant $H_{\mathrm{a}}$, panels I-L). As in the previous figure solid lines show results for scenarios with maternal investment ('mother pays') and dashed lines without ('offspring pay'). Black curves always indicate scenarios in which the focal parameter value was halved and grey curves scenarios in which the value was doubled. The characteristic patterns shown before, i.e. bimodal and fat-tailed kernels for maternal investment, are stable. Wide kernels lead to larger dispersal distances (A, B), a more pronounced bimodality (C) and extremely fat tails (D). Bimodality emerges also with very narrow competition kernels, yet requires higher dispersal costs. More platykurtic competition kernels tend to underline the described effects, while leptokurtic competition kernels do not lead to a loss of fat tails or bimodality $(\mathrm{E}-\mathrm{H})$. Slow growth reduces dispersal distances and underlines the differences between scenarios with and without maternal investment (bimodality, K). Parameters: $\lambda_{0}=4, \mu_{0}=0.1, \sigma=0.5$ (narrow) or $\sigma=2$ (wide), $\gamma=1$ (leptokurtic) or $\gamma=4$ (platykurtic), and $H_{\mathrm{a}}=1.5$ (fast growth) or $H_{\mathrm{a}}=6$ (slow growth). The lines are smooth spline regressions (smoothing parameter: $\lambda=0.3)$ 


\section{Tables and Figures}

Table 1

\begin{tabular}{lcl}
\hline parameter & values & meaning \\
\hline$\lambda_{0}$ & $2,4,8$ & fecundity \\
$\mu_{0}$ & $0.05,0.1,0.2$ & baseline mortality (density independent) \\
$\sigma$ & $0.5,1,2$ & width of the competition kernel (standard deviation) \\
$\gamma$ & $1,2,4$ & kurtosis of competition kernel \\
$H_{a}$ & $1.5,3,6$ & age-dependence of competition (half-saturation constant) \\
$\mu_{d}^{0}$ & $0.05,0.1,0.15, \ldots, 0.4$ & dispersal costs (per distance unit) \\
\hline
\end{tabular}




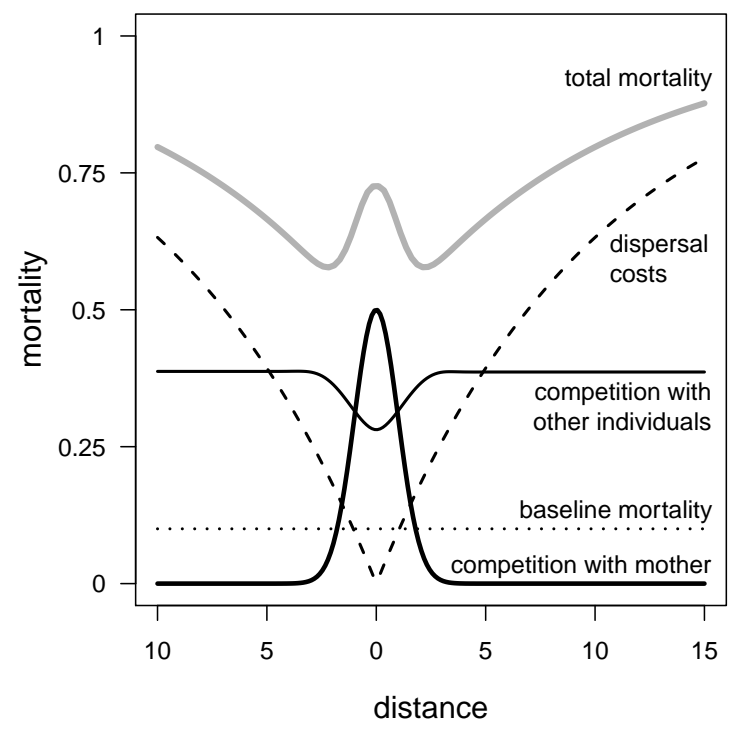

Figure 1 


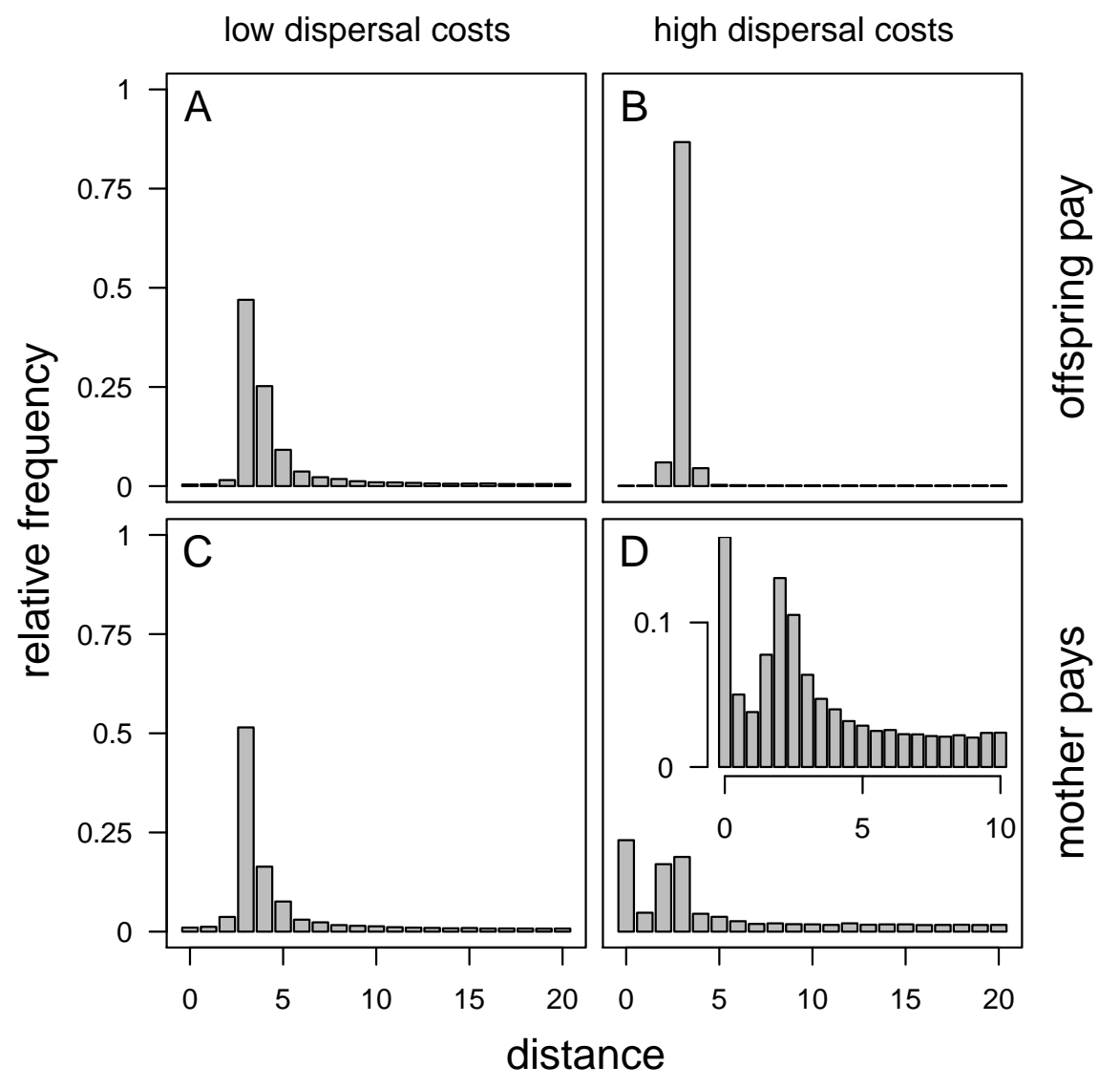

Figure 2 

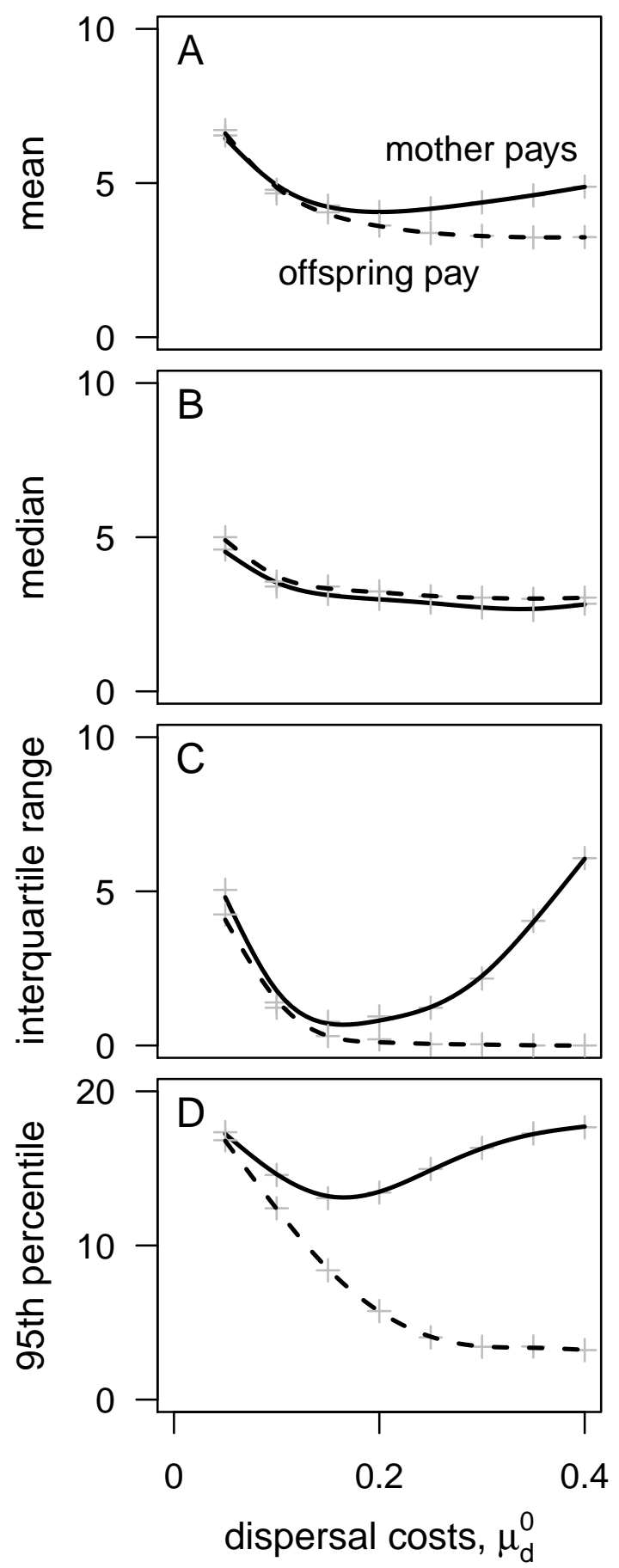

Figure 3 


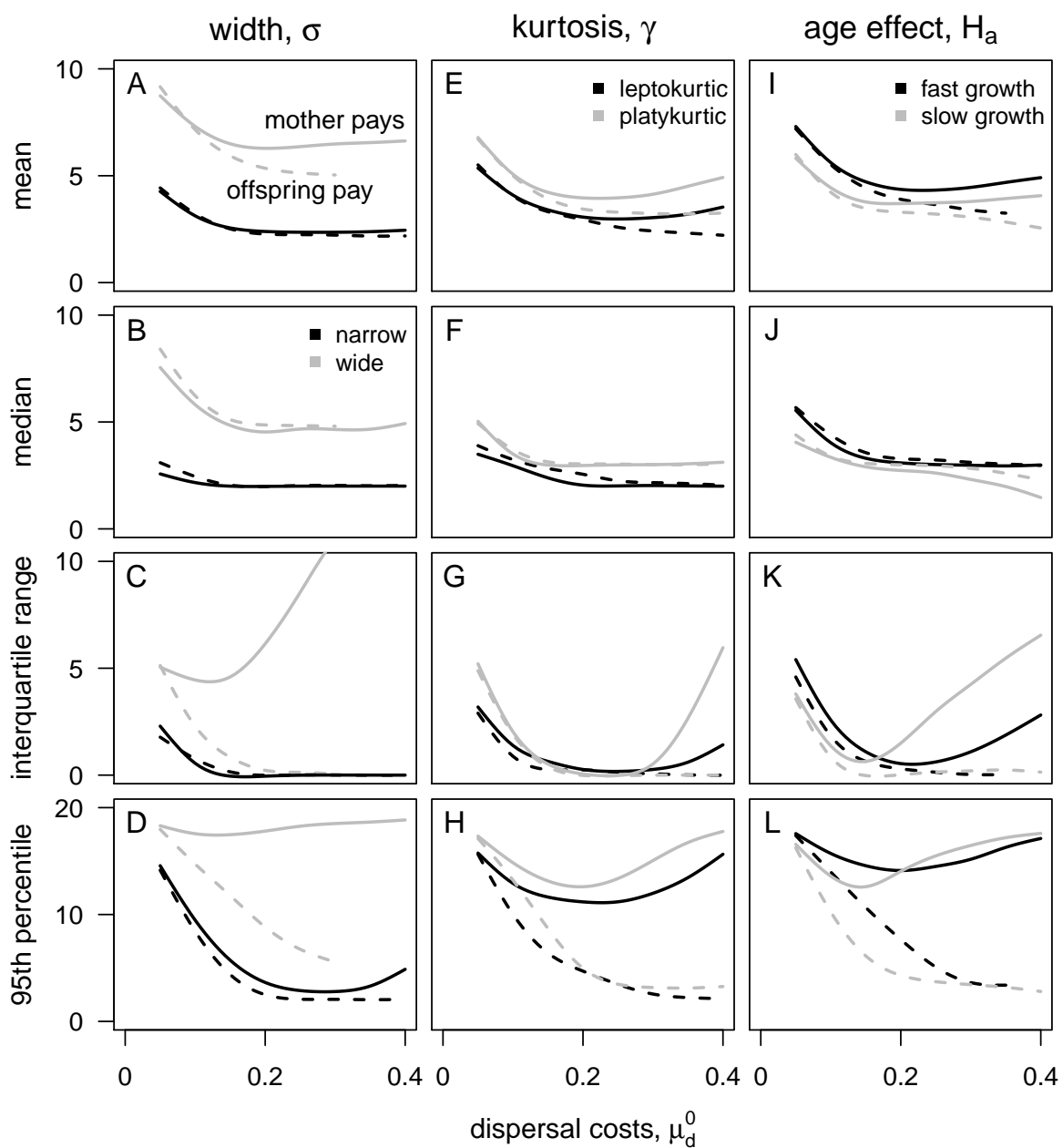

Figure 4 


\section{- Supporting Information}

2 Fronhofer, E.A., Poethke, H.J. and Dieckmann, U.: Evolution of dispersal distance: maternal investment leads to bimodal dispersal kernels

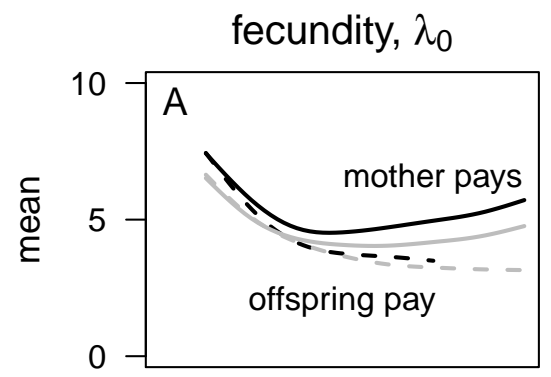

baseline costs, $\mu_{0}$
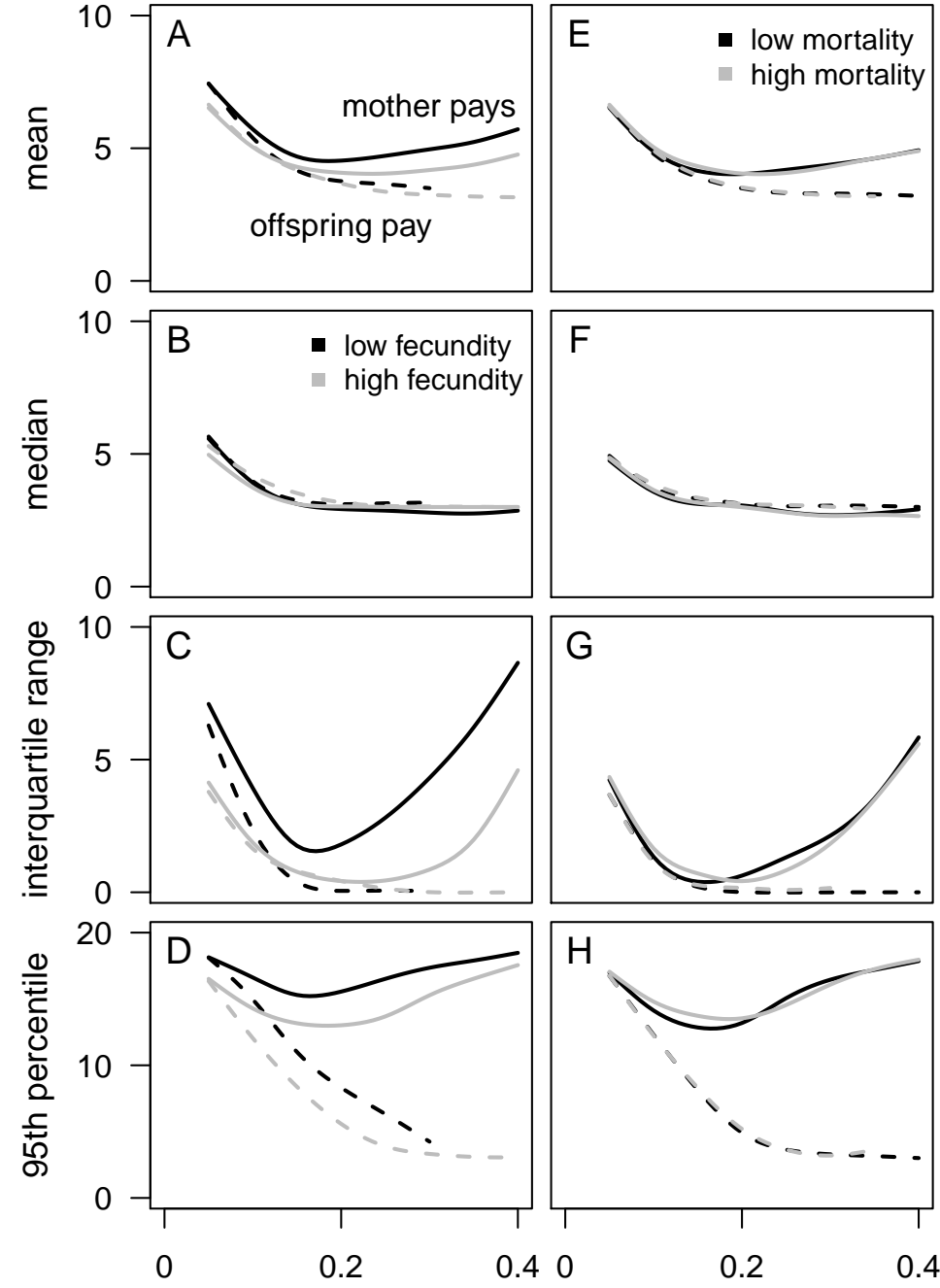

dispersal costs, $\mu_{d}^{0}$

Figure S1: Influence of fecundity and mortality. Solid lines show results for scenarios with maternal investment ('mother pays') and dashed lines without ('offspring pay'). Black curves always indicate scenarios in which the focal parameter value was halved and grey curves scenarios in which the value was doubled. Low fecundities $\left(\lambda_{0}\right)$ underline the effects described above $(\mathrm{A}-\mathrm{D})$, but higher fecundities do not destroy the patterns, i.e., fat tails and bimodality for maternal investment still arise at sufficiently high dispersal costs. Density independent baseline mortality $\left(\mu_{0} ; \mathrm{E}-\mathrm{H}\right)$ does not influence our results in a quantitatively relevant way. Parameters: $\gamma=2, \sigma=1, H_{\mathrm{a}}=3, \lambda_{0}=2$ (low fecundity) or $\lambda_{0}=8$ (high fecundity), and $\mu_{0}=0.05$ (low mortality) or $\mu_{0}=0.2$ (high mortality). The lines are smooth spline regressions (smoothing parameter: $\lambda=0.3$ ). 


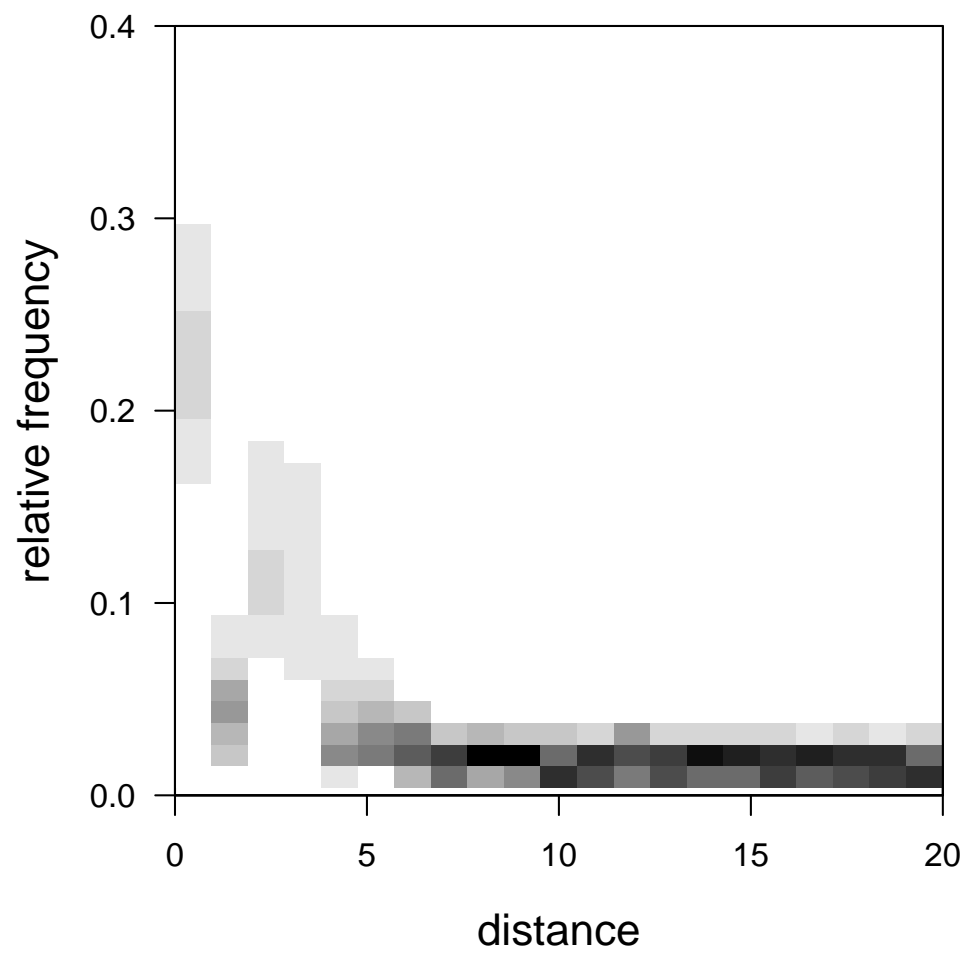

Figure S2: Evolutionarily stable dispersal kernel as a 2D histogram. This figure corresponds to figure $2 \mathrm{D}$ and shows the strategies of all individuals across all 25 replicate simulation runs. Clearly, the bimodality occurs at the individual kernel level and is not a result of a mixed strategy at population level. Parameters: $\lambda_{0}=4, \mu_{0}=0.1, \gamma=2, \sigma=1, H_{\mathrm{a}}=3$, and $\mu_{\mathrm{d}}^{0}=0.4$. 


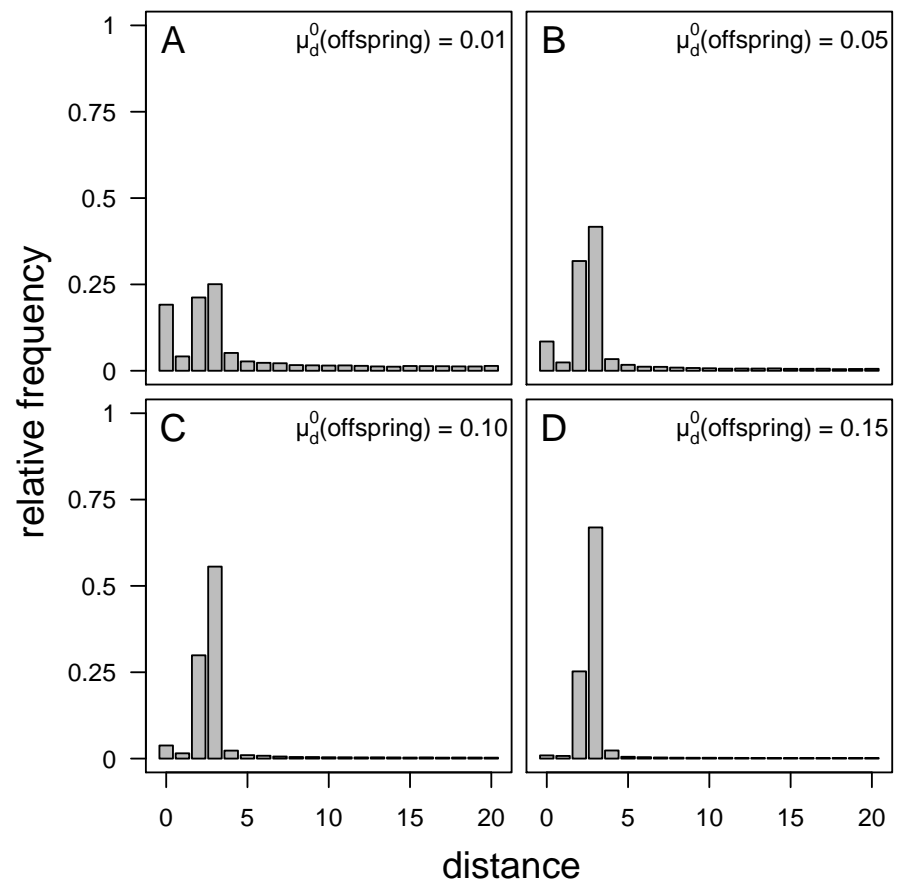

Figure S3: Maternal investment and the evolution of dispersal kernels. All four panels show evolutionarily stable dispersal kernels (distance pdfs) for scenarios with maternal investment, i.e. the mother pays kerneldependent dispersal costs. Additionally, different level of direct dispersal costs paid by the offspring are included. These results show that if both, mother and offspring, have to pay dispersal costs our results hold up to a considerable amount of costs paid by both. Parameters: $\lambda_{0}=4, \mu_{0}=0.1, \gamma=2, \sigma=1$, $H_{\mathrm{a}}=3$, and $\mu_{\mathrm{d}}^{0}=0.4$ for the mother. 


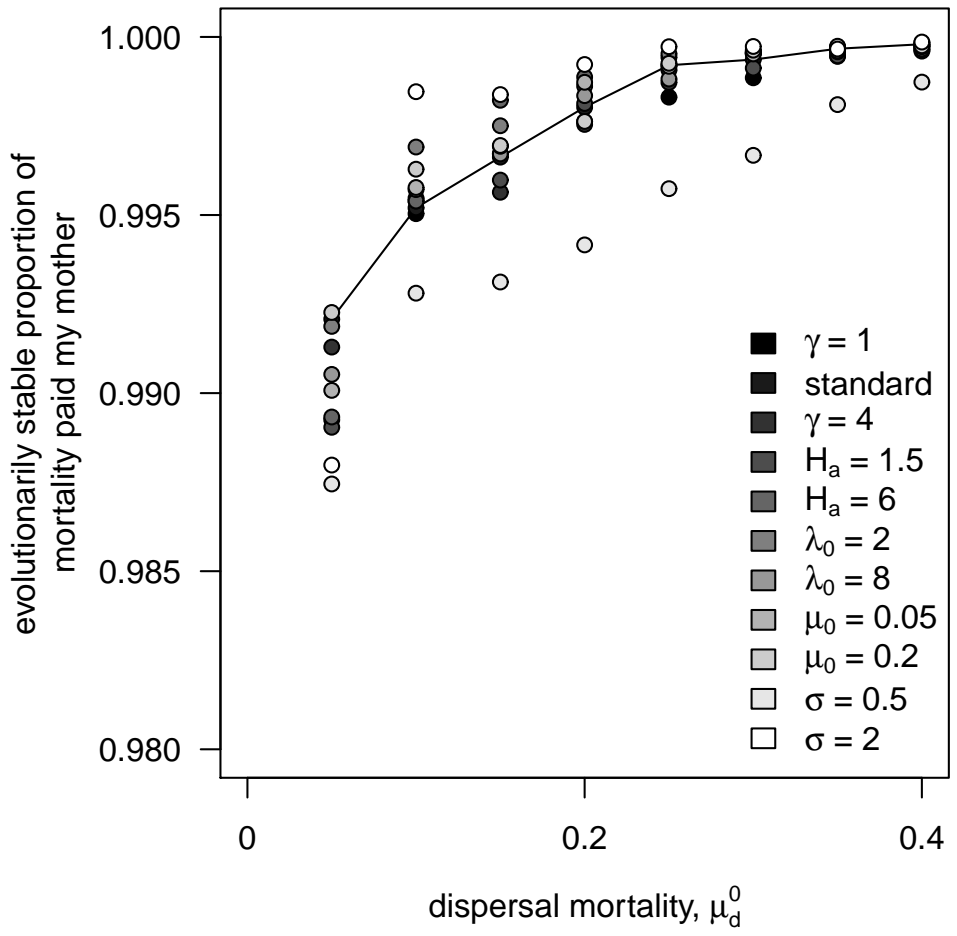

Figure S4: Evolutionarily stable allocation of dispersal costs to mother vs. offspring. We here allowed the allocation of costs to be itself an evolvable trait: depending on this trait a proportion of the distance dependent dispersal costs $\left(\mu_{\mathrm{d}}^{0}\right)$ is paid by the mother and the remaining costs are paid by the offspring. For all tested parameter combinations (table 1) no intermediate cost allocation strategy evolved and the evolutionarily stable strategy was full maternal investment, i.e. 'mother pays'. Note the scale of the y-axis. The line corresponds to the our standard parameter combination. 


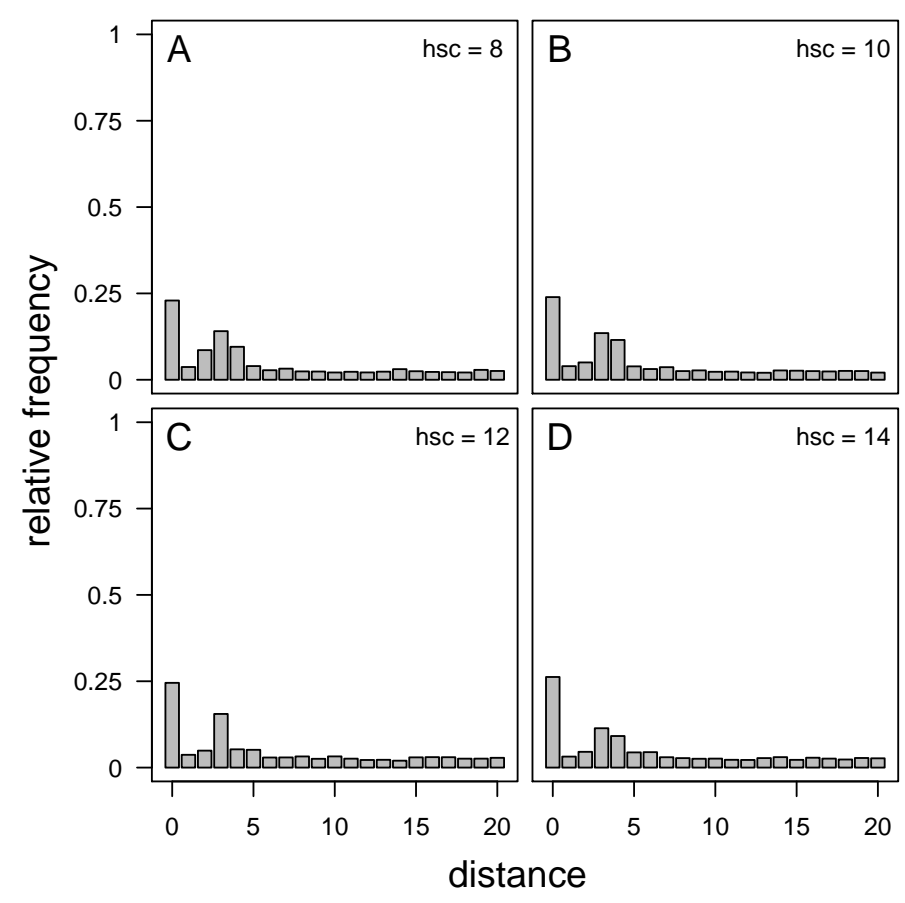

Figure S5: Maternal investment, baseline mortality - fertility trade-off, and the evolution of dispersal kernels. All four panels show evolutionarily stable dispersal kernels (distance pdfs) for scenarios with maternal investment, a trade-off between baseline mortality and fecundity and evolving fecundities. The trade-off function is saturating and follows the general form $\mu_{0}=\lambda_{0} /\left(\lambda_{0}+h s c\right)$ (see figure S6). Clearly, bimodality is not affected by the inclusion of this additional trade-off. Parameters: $\lambda_{0}=$ evolving, $\mu_{0}=0.1, \gamma=2, \sigma=1, H_{\mathrm{a}}=3$, and $\mu_{\mathrm{d}}^{0}=0.4$. 


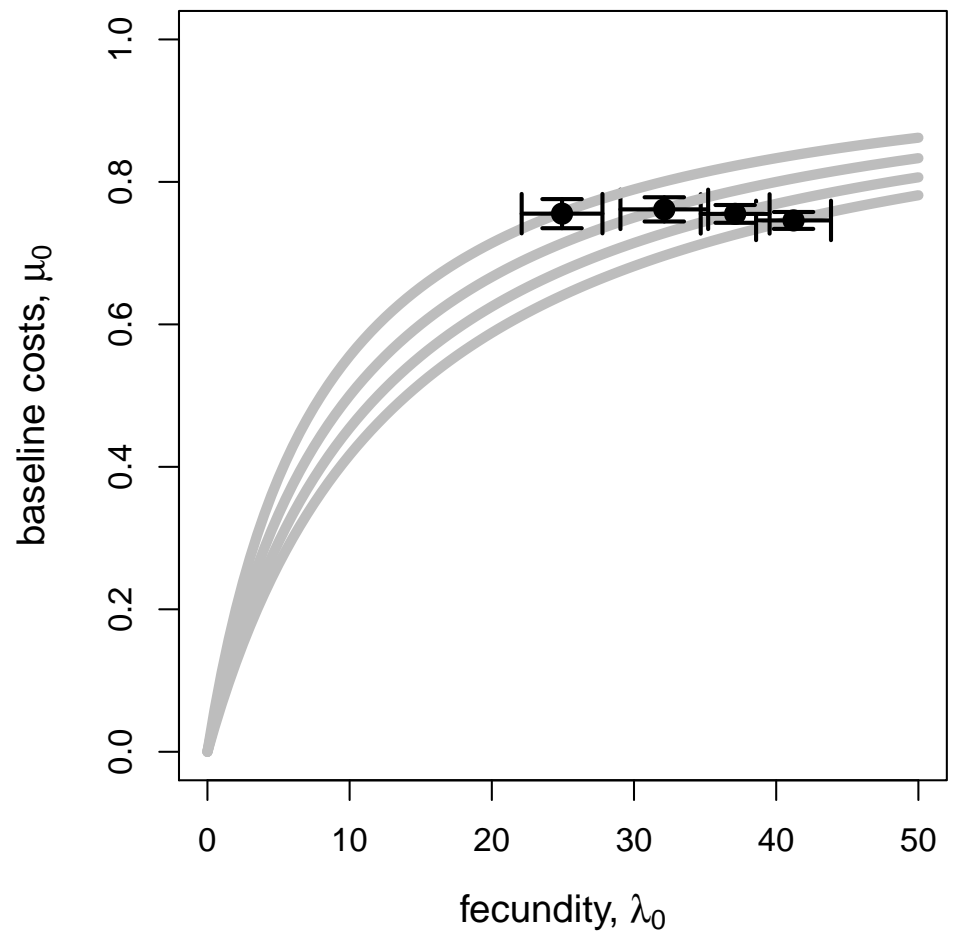

Figure S6: Maternal investment, baseline mortality - fertility trade-off, and the evolution of dispersal kernels. The figure shows the evolutionarily stable fertility (mean and standard deviation) and the corresponding baseline mortality for scenarios with maternal investment and a trade-off between baseline mortality and fecundity. The grey lines depict a sample of tested trade-off functions (from top to bottom: $h s c=8,10,12,14)$. Here, the trade-off function is saturating and follows the general form $\mu_{0}=\lambda_{0} /\left(\lambda_{0}+\right.$ hsc). Similar results were obtained for linear and concave functions. Such trade-offs lead to highly fertile annual organisms with strongly bimodal dispersal kernels (figure S5). Parameters: $\lambda_{0}=e v o l v i n g$, $\mu_{0}=0.1, \gamma=2, \sigma=1, H_{\mathrm{a}}=3$, and $\mu_{\mathrm{d}}^{0}=0.4$. 